\title{
Evaluación de los servicios ecosistémicos provistos por una microcuenca periurbana de Querétaro (México).
}

\author{
Assessment of ecosystem services provided by a periurban basin of Queretaro (Mexico).
}

Historial del artículo

Recibido:

11 de junio de 2019.

Aceptado:

05 de julio de 2019.

\section{Dora Beatriz Palma Hernández $z^{\mathrm{a}}$, Francisco de la Barrera ${ }^{\mathrm{b}}$, Raúl Pineda López ${ }^{\mathrm{a}}$}

a Maestría en Gestión Integrada de Cuencas, Universidad Autónoma de Querétaro (UAQ), México.

${ }^{b}$ Facultad de Arquitectura, Urbanismo y Geografía, Universidad de Concepción y Centro de Desarrollo Urbano Sustentable (CEDEUS), Chile. Correo electrónico: fdelabarrera@gmail.com

\section{Palabras clave}

Mapeo de servicios ecosistémicos, oferta de servicios ecosistémicos, SIG

\section{Keywords}

GIS, mapping of ecosystem services, supply of ecosystem services

\begin{abstract}
Resumen
Las actividades humanas tienen efectos en el medio ambiente y en la capacidad de los ecosistemas para proporcionar servicios ecosistémicos. En esta investigación se evalúan y mapean los servicios ecosistémicos de una microcuenca periurbana de Querétaro, con el fin de generar un panorama de la oferta potencial de servicios ecosistémicos y su relación con la demanda que podría haber de parte de los habitantes de la microcuenca. Se analizaron quince servicios ecosistémicos locales que podrían permitir a los tomadores de decisiones implementar medidas a corto y mediano plazo en temas como la gestión del riesgo y adaptación al cambio climático. La evaluación se basa en una evaluación basada en conocimiento de expertos y la aplicación de una encuesta para reconocer los servicios más demandados. La evaluación biofísica realizada sigue un esquema basado en la capacidad de los ecosistemas de proveer estos servicios. Se analizaron ocho tipos de ecosistemas de la microcuenca para evaluar todos los servicios de manera general y luego se seleccionaron cuatro servicios, representativos de cada tipo de servicio (provisión, regulación y cultural) y relevantes a nivel local para mapearlos por medio de SIG. Los resultados muestran las diferentes capacidades que tienen los ecosistemas de la microcuenca El Nabo para proveer servicios ecosistémicos, y a pesar de ser mayor la oferta que la demanda de algunos servicios ecosistémicos en el área con ecosistemas naturales, la zona urbana tiene un alto déficit de servicios y este excedente de oferta es insuficiente para cubrir las necesidades que los habitantes demandan. Contar con mapas de oferta, demanda y balance potencial de servicios ecosistémicos representa una contribución importante para aplicaciones futuras y servir como base para la propuesta de pago por servicios ecosistémicos que se discute en México.
\end{abstract}

\begin{abstract}
Human activities have an impact on the environment and on the capacity of ecosystems to provide ecosystem services. In this research, ecosystem services of a periurban microbasin of Querétaro are evaluated and mapped in order to generate a panorama of the potential supply and its association with the demand that inhabitants could have. Fifteen local ecosystem services were analysed. For decision makers they could allow to implement shortand medium-term measures in topics such as risk management and adaptation to climate change. The biophysical evaluation carried out is based on an evaluation based on expert knowledge and the application of a survey to recognize the most demanded services. Eight types of ecosystems were analyzed to evaluate all the ecosystem services in a general way. Then, four local ecosystem services were selected, representative of each type of service (provision, regulation and cultural) to map them through GIS. The results show the different capacities that the ecosystems of the El Nabo microbasin have to provide ecosystem services, and in spite of the fact that the supply of some ecosystem services in the area with natural ecosystems is greater than the demand, the urban area has a high deficit of services and this surplus of supply is insufficient to cover the needs demanded by the inhabitants. Having maps of potential supply, demand and balance of the ecosystem services represents an important contribution for future applications and serves as a basis for the proposal for payments for ecosystem services that is being discussed in México.
\end{abstract}




\section{Introducción}

Los servicios ecosistémicos (SE) representa una aproximación conceptual para reconocer explícitamente los beneficios que los ecosistemas generan para el bienestar de las personas, (Daily, 1997; MEA, 2005; Sukhdev, Wittmer $\&$ Miller, 2014). Desde que el concepto fue propuesto se han generado diversos esfuerzos para listar cuales son los beneficios, mediante clasificaciones como las propuestas por Costanza et al. (1997), De Groot, Wilson \& Boumans (2002), MEA (2005), Wallace (2007) y Turner, Fisher \& Zylstra (2008). En general estas coinciden en generar tipologías y luego servicios específicos, la última de las clasificaciones, y que ha sido ampliamente adaptada de manera oficial por gobiernos, se deriva de la Agencia Ambiental Europea (European Enviroment Agency, 2019), quien lidera la denominada "Clasificación común internacional de Servicios Ecosistémicos" (CICES), de acuerdo a la cual existen tres tipos de SE: 1) provisión, 2) regulación y mantención, 3) culturales, y 75 servicios específicos (Haines-Young \& Potschin, 2011). En Latinoamérica, los estudios sobre SE se han acelerado notablemente, especialmente por la importancia de la biodiversidad en el continente y su grado de amenaza, siendo los ecosistemas asociados a áreas urbanas menos analizados, dominando enfoques economicistas (Balvanera \& Cotler, 2007; Balvanera et al., 2012; Delgado \& Marín, 2015; Dobbs et al., 2019; Potschin \& Haines-Young, 2011).

En las microcuencas periurbanas los SE juegan un papel importante en las decisiones sobre el aprovechamiento de los recursos naturales, obteniendo beneficios de uso directo como la producción de alimentos, provisión y regulación de agua, control de inundación, erosión, pestes y plagas, entre otros. Asimismo existen beneficios de uso indirecto, los cuales se relacionan con procesos ecosistémicos como el ciclo de nutrientes, y valores culturales, espirituales y estéticos. En Latinoamérica, las microcuencas periurbanas se caracterizan por la alta ocupación humana, existiendo sobreexplotación de los recursos naturales y un aprovechamiento que no es sustentable ni eficiente, sin consideración por los efectos adversos, ni por la alteración del ecosistema, lo que provoca problemas ambientales serios. El marco conceptual de los servicios ecosistémicos es útil ante fenómenos como éste, dado que aporta información para evaluar y valorar el rol de los ecosistemas y la biodiversidad para la provisión de beneficios, siendo objeto de una atención cada vez mayor (Balvanera et al., 2012; Carpenter et al., 2009; De la Barrera, Bachman-Vargas y Tironi 2015; De Groot, Alkemade, Braat, Hein \&Willemen, 2010; Dobbs et al., 2019).
Los servicios que una microcuenca provee representan un valor económico diferente para cada uno de los actores involucrados. En la parte alta y media de la cuenca se limita o favorece la oferta de SE, mientras que cuenca abajo están quienes más requieren los SE y podrían estar dispuestos a pagar por la generación de un servicio de calidad (Barzev, 2000). No obstante, frecuentemente los habitantes utilizan los recursos de manera descontrolada, generando desequilibrios ecológicos que afectan la disponibilidad de éstos (Santillán, 2013). Se ha señalado, que la falta de mecanismos de valoración de los SE provoca que no exista una estimación económica que refleje cuánto cuesta producirlos, ni cuántos SE son producidos, limitando el desarrollo de alternativas de manejo que aseguren la sustentabilidad del territorio (Torres \& Guevara, 2002). La valoración de servicios ecosistémicos se puede hacer desde diversas perspectivas: la biofísica, que hace una estimación o cuantificación física y/o ecológica de los SE; social, donde se registra la percepción sociocultural del bienestar brindado por los ecosistemas; y la económica, que remite a cuantificación o estimación monetaria o transaccional del servicio. En particular, en este trabajo se usa un método biofísico que se ha implementado en distintas partes del mundo para valorar los SE, y consiste en mapear la oferta potencial, la demanda potencial y su balance potencial (Burkhard, Kroll, Müller \& Windhorst, 2009). Su uso sirve para implementar un enfoque sistémico en la toma de decisiones en políticas públicas y para la planificación territorial, de manera de maximizar la oferta de SE e integre dimensiones ecológicas y sociales con la vulnerabilidad ante la pérdida de SE, lo que podría ser un paso importante para el desarrollo de políticas públicas ambientales en Latinoamérica (Laterra, Barral, Carmona \& Nahuelhual, 2016).

Este trabajo se enfoca en Querétaro, que entre 2000 y 2018 ha tenido una tasa de crecimiento poblacional medio anual de $1,64 \%$, convirtiéndolo en una zona metropolitana con más de un millón de habitantes (CONAPO, 2018). De acuerdo a Rosas (2014), en la ciudad de Querétaro, existen alrededor de 400 mil hectáreas capaces de brindar SE y solamente 90 mil reciben un subsidio para asegurarlo (Rosas, 2014). La Zona Occidental de Microcuencas (ZOM) del Municipio de Querétaro fue decretada en 2006 como área natural protegida (ANP) de competencia local y contempla siete microcuencas que corresponden al $45 \%$ de las áreas de muy alta infiltración del Municipio de Querétaro, teniendo una riqueza de flora importante que brinda servicios ecosistémicos (CONACYT, 2014). La microcuenca El Nabo es una de estas siete microcuencas (Figura 1) y es la más pequeña en extensión del territorio mencionado. Sin embargo, debido a su ubicación periurbana 
y cobertura de vegetación, se considera como la zona más conservada de toda el ANP.

Este trabajo busca aportar en la discusión que existe en México sobre formas de valorar los servicios ecosistémicos y en particular al esquema de Pago por Servicios Ambientales (PSA), aplicando una metodología sencilla que permita evaluar y cartografiar la oferta, demanda y balance potencial de servicios ecosistémicos en una microcuenca periurbana de Querétaro (El Nabo) y que sea replicable en otras microcuencas. En esta microcuenca no se han hecho hasta la fecha evaluaciones de SE, por lo que este trabajo pretender también dar soporte a la valoración del rol de sus ecosistemas y servir para incentivar la existencia de incentivo económico a las comunidades de la microcuenca que opten por para proteger y preservar los recursos naturales.

\section{Materiales y métodos}

\section{Zona de estudio}

La microcuenca El Nabo se localiza en la parte centronorte del municipio de Querétaro (342139, 2291259 UTM, huso14N) (Figura 1). Cuenta con una superficie de 26,28 km2, una elevación media de $2.100 \mathrm{msnm}$, una precipitación media anual de $540 \mathrm{~mm}$ y un $52 \%$ de cobertura vegetal compuesta por bosque, matorral y pastizal. De acuerdo con la clasificación de Köppen-García, el clima de la microcuenca se clasifica como semiseco templado en la parta alta y semiseco cálido en la parte baja. Incluye una diversidad de ecosistemas naturales correspondientes a cuatro tipos de cobertura vegetal: bosque tropical caducifolio, matorral crasicaule, matorral subtropical y pastizal (Pineda, Domínguez, Hernández y Ventura, 2005) y una intensa actividad agrícola y urbana (Figura 2).

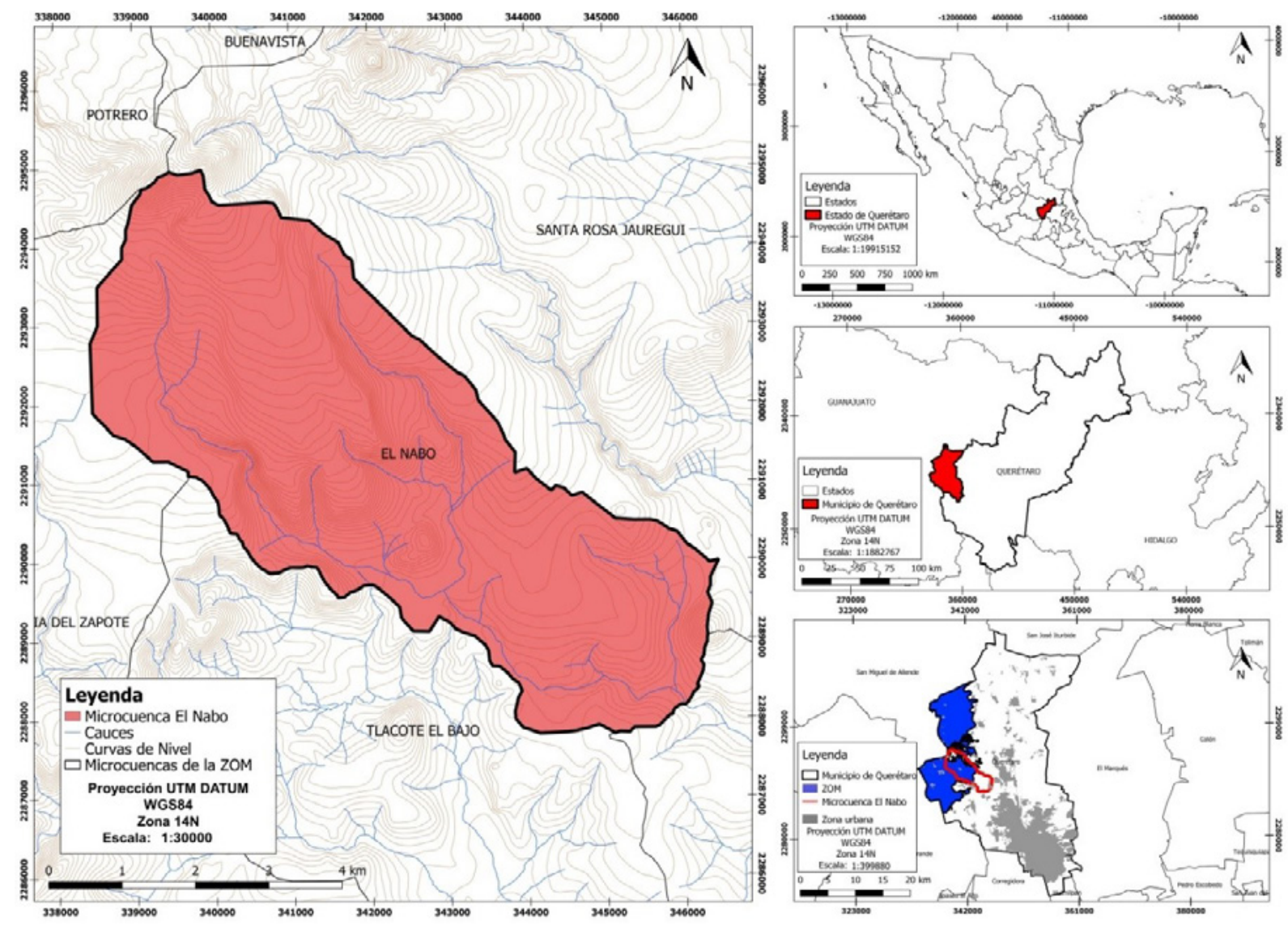

Figura 1. Ubicación de la microcuenca El Nabo, Querétaro. Fuente: Elaboración propia a partir de datos de INEGI (2015). 


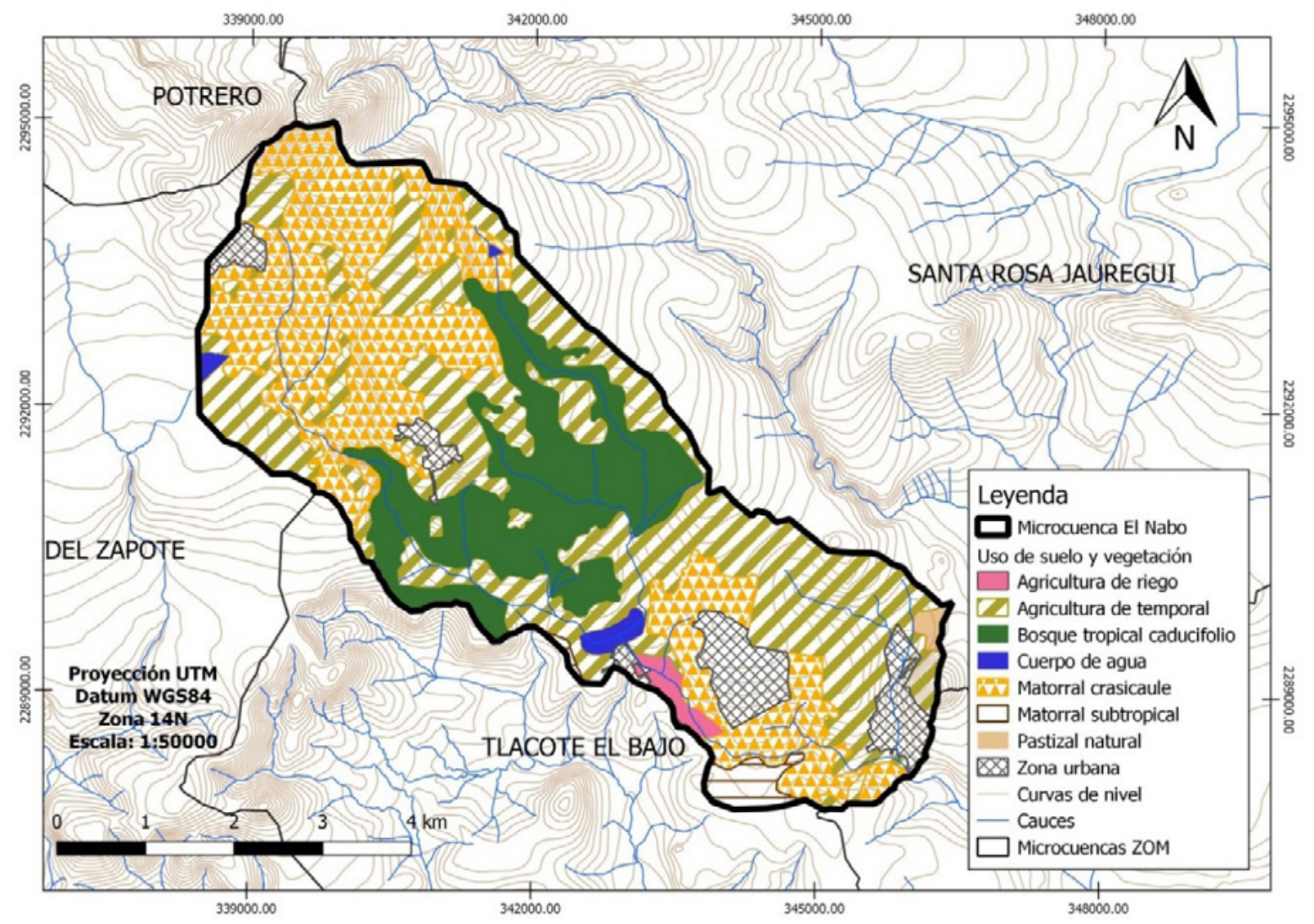

Figura 2. Ecosistemas representados a través de los usos y coberturas del suelo y vegetación de la microcuenca El Nabo. Fuente: Elaboración propia con información del Inventario Forestal Municipal de Querétaro (2017).

El estudio está basado en un esquema de evaluación biofísica, no monetaria, ocupando el método de mapeo de suministro de servicios ecosistémicos propuesta por Burkhard et al. (2009), así como también el mapeo de la demanda y balance potencial de servicios ecosistémicos (Burkard, Kroll, Nedkov \& Müller, 2012), que ha sido implementado en distintos trabajos en el mundo y en Latinoamérica (Arriagada \& Aguayo, 2018; Burkhard, Kandziora, Hou \& Müller, 2014; Kopperoinen, Itkonen \& Niemelâ, 2014; Koschke, Fuerst, Frank \& Makeschin, 2012; Montoya-Tangarife, De La Barrera, Salazar e Inostroza, 2017; Vihervaara, Kumpula, Tanskanen \& Burkhard, 2010). Esta metodología consiste en que expertos asignan valores en matrices de oferta y demanda de SE y luego esos valores son sistematizados en una matriz única, lo que en este caso hizo uno de los autores generando consenso entre los expertos (otros autores de este trabajo y otros investigadores). Para espacializar los tipos de ecosistemas se utilizó como proxy la base de datos de usos del suelo y vegetación del Inventario Forestal Municipal de Querétaro 2017, escala 1:20.000 (Figura 2), cuyas principales características se presentan en la Tabla 1. La selección de quince SE con potencial en la zona, se basó en aquellos SE locales que permitan a los tomadores de decisiones implementar medidas a corto y mediano plazo en temas como la gestión del riesgo y adaptación al cambio climático.

\section{Oferta potencial de servicios ecosistémicos mediante consulta a expertos}

Para construir la matriz de oferta de SE se usaron los 15 servicios ecosistémicos con potencial en la zona (en columnas) y los 8 tipos de usos de suelo y vegetación del Inventario Forestal Municipal de Querétaro presentado en la Tabla 1 (en filas). En las intersecciones, se evaluaron de forma cualitativa las capacidades de oferta potencial de los diferentes ecosistemas para proporcionar SE en 
Tabla 1

Características principales en los tipos de usos del suelo vegetación como proxies de los ecosistemas dela microcuenca El Nabo.

\begin{tabular}{|c|c|}
\hline $\begin{array}{l}\text { Tipos de ecosistemas o usos de } \\
\text { suelo y vegetación }\end{array}$ & Descripción \\
\hline Agricultura de temporal & $\begin{array}{l}\text { Uso agrícola donde, con una periodicidad marcada, se producen alimentos como maiz, frijol, sorgo, cebada, } \\
\text { avena, alfalfa. Se encuentra en todas las zonas de la microcuenca (alta, media y baja) y ocupa 10,86 km2 } \\
\text { que corresponde al } 41 \% \text { de la superficie total de la microcuenca. }\end{array}$ \\
\hline Agricultura de riego & $\begin{array}{l}\text { Uso agrícola que depende de la irrigación y que ocupa } 0,33 \mathrm{~km} 2 \text { lo que corresponde a sólo un 1,3\% de la } \\
\text { cuenca. Se produce principalmente chile. }\end{array}$ \\
\hline Bosque tropical caducifolio & $\begin{array}{l}\text { Ecosistema natural cuya cobertura se encuentra sobre los } 1.750 \text { m.s.n.m. en cañadas y lugares protegidos o } \\
\text { laderas pronunciadas asociadas a cauces de ríos sobre suelos someros. Su cobertura está conformada por } \\
\text { árboles de } 6-8 \mathrm{~m} \text { de alto, compuesta principalmente por Lysiloma microphyla, Bursera fagaroides, B. palmeri, } \\
\text { entre otras. Ocupa } 5,04 \mathrm{~km} 2 \text { que corresponden al } 19 \% \text { de la microcuenca. }\end{array}$ \\
\hline Matorral crasicaule & $\begin{array}{l}\text { Cobertura vegetal natural que se encuentra en la zona alta y baja de la cuenca, en laderas pronunciadas, } \\
\text { con suelos muy pedregosos, con arbustos y plantas arborescentes de } 2-4 \mathrm{~m} \text {. Predominan especies como } \\
\text { Myrtillocactus geometrizans, Stenocereus dumortieri, Opuntia spp. También se presenta elementos leñosos } \\
\text { como Acacia schaffneri, A. farnesiana, Ipomoea murucoides, Karwinskia humboldtiana, Condalia mexicana y } \\
\text { C. velutina. Ocupa } 8,25 \mathrm{~km} 2 \text { lo que corresponde al } 31 \% \text { de la microcuenca }\end{array}$ \\
\hline Matorral subtropical & $\begin{array}{l}\text { Cobertura vegetal natural que se distribuye en la zona baja de la microcuenca, sobre planicies o valles con } \\
\text { suelos de aluvión. Presenta árboles y arbustos con espinas de hasta } 5 \mathrm{~m} \text { de alto, principalmente Prosopis } \\
\text { laevigata, A. farnesiana, A. schaffneri, Mimosa acauleticarpa, Celtis pallida y Opuntia imbricata. Ocupa } 0.44 \\
\mathrm{~km} 2 \text { lo que corresponde solo a un } 1,7 \% \text { de la microcuenca. }\end{array}$ \\
\hline Pastizal natural & $\begin{array}{l}\text { Cobertura vegetal que se produce por la actividad pecuaria, para ganado bovino y caprino. La mayoría de } \\
\text { las especies son herbáceas. Presenta especies como Ipomoea capillacea, Jatropha dioica, Aristida sp. entre } \\
\text { otras. Cubren solo el 0,4\% de la cuenca. }\end{array}$ \\
\hline Cuerpos de agua & Cobertura del suelo que incorpora a presas, lagunas, arroyos. Ocupa el 0,93\% de la microcuenca \\
\hline Área urbana & Uso del suelo que se concentra principalmente al sureste de la microcuenca. \\
\hline
\end{tabular}

Fuente: Inventario Forestal Municipal de Querétaro (2017).

una escala donde: $0=$ no hay capacidad relevante; $1=$ capacidad relevante baja; $2=$ capacidad relevante; $3=$ capacidad relevante media, $4=$ capacidad relevante alta y $5=$ capacidad relevante muy alta. Debido a que no existen estudios previos sobre la valoración de SE del lugar, el llenado se llevó a cabo por expertos del área de ingeniería ambiental, geografía, cuencas y ecología urbana y del paisaje, teniendo en consideración conocimiento del lugar, del funcionamiento ecosistémico y de las características geográficas de la cuenca.

\section{Demanda potencial de servicios ecosistémicos mediante consulta a expertos y encuesta a habitantes}

Para evaluar la demanda se utilizaron los mismos servicios ecosistémicos y ecosistemas de la oferta potencial y se analizaron datos estadísticos y censos de población (INEGI, 2010), con el fin de tener información demográfica y de la distribución de la población. Se elaboró una matriz similar a la de oferta, que muestra hipótesis iniciales sobre la demanda potencial de servicios ecosistémicos. Los valores en la matriz de demanda seis rangos cualitativos, siguiendo la propuesta original de Burkhard et al. (2009), donde cada valor representa lo siguiente: $0=$ ninguna demanda relevante; $1=$ baja demanda relevante; $2=$ demanda relevante; $3=$ demanda relevante media; $4=$ alta demanda relevante y $5=$ demanda relevante muy alta. Así como la matriz de oferta, se llenó teniendo conocimiento del lugar, la cantidad de personas, ocupaciones, necesidades $\mathrm{y}$ el uso que le dan a los SE.

Estos valores fueron complementados con el desarrollo de una encuesta de 23 preguntas, que se aplicó a la población que potencialmente es beneficiada por los $\mathrm{SE}$ de la microcuenca. La encuesta fue aplicada por medio de la plataforma Survey Monkey y contó con información que 
sirviera como capacitación dirigida a representantes locales de colonias. La información consideró datos relevantes sobre los ecosistemas y sus SE, la modificación de éstos y características socioeconómicas. La aplicación de la encuesta permitió determinar los SE más demandados y disponibilidad a pagar para acceder a esos servicios, mediante el método de valoración contingente, de modo de complementar los aportes al esquema de PSA.

\section{Balance entre oferta y demanda potencial de servicios ecosistémicos}

Finalmente, para determinar si existe balance entre la oferta y demanda de SE, se utilizó la información de las matrices en donde la oferta potencial fue restada a la demanda potencial y se obtuvo una nueva matriz con el balance potencial de servicios ecosistémicos en los ecosistemas de El Nabo. El resultado tiene una escala que varía entre $-5=$ donde la demanda excede significativamente la oferta o el suministro es insuficiente; a 0 si demanda $=$ oferta; y a 5 donde la oferta supera la demanda significativamente o hay un exceso de oferta.

\section{Resultados}

\section{Oferta potencial de SE}

La tabla 2 revela las capacidades de los ecosistemas para proveer servicios ecosistémicos. Se puede observar que los ecosistemas modificados por el hombre tienen

Tabla 2

Matriz de evaluación de las capacidades de oferta de servicios ecosistémicos de los ecosistemas de la microcuenca El Nabo.

\begin{tabular}{|c|c|c|c|c|c|c|c|c|c|c|c|c|c|c|c|}
\hline \multicolumn{16}{|c|}{ OFERTA POTENCIAL } \\
\hline & \multicolumn{5}{|c|}{ Servicios ecosistémicos de Provisión } & \multicolumn{5}{|c|}{ Servicios ecosistémicos de Regulación } & \multicolumn{5}{|c|}{ Servicios ecosistémicos Culturales } \\
\hline $\begin{array}{l}\text { Usos de suelo } \\
\text { y vegetación } \\
\text { (ECOSISTEMAS) }\end{array}$ & 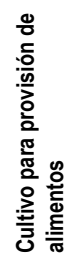 & 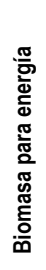 & 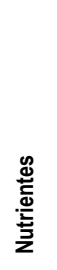 & 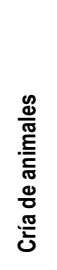 & 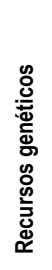 & 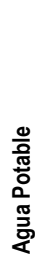 & 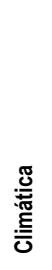 & 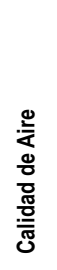 & 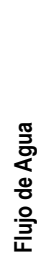 & 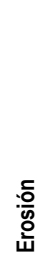 & 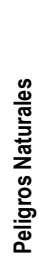 & 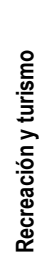 & 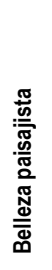 & 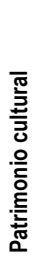 & 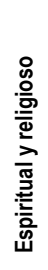 \\
\hline $\begin{array}{l}\text { Agricultura de } \\
\text { temporal }\end{array}$ & 4 & 3 & 3 & 2 & 1 & 0 & 2 & 1 & 2 & 3 & 1 & 3 & 3 & 4 & 4 \\
\hline $\begin{array}{l}\text { Agricultura de } \\
\text { riego }\end{array}$ & 3 & 2 & 2 & 2 & 2 & 0 & 1 & 1 & 2 & 2 & 1 & 2 & 3 & 3 & 4 \\
\hline $\begin{array}{l}\text { Bosque tropical } \\
\text { caducifolio }\end{array}$ & 1 & 5 & 4 & 3 & 4 & 0 & 5 & 5 & 5 & 5 & 5 & 5 & 5 & 4 & 4 \\
\hline $\begin{array}{l}\text { Matorral } \\
\text { crasicaule }\end{array}$ & 2 & 5 & 5 & 4 & 5 & 0 & 5 & 5 & 5 & 4 & 4 & 5 & 5 & 5 & 5 \\
\hline $\begin{array}{l}\text { Matorral } \\
\text { subtropical }\end{array}$ & 2 & 5 & 4 & 5 & 4 & 0 & 4 & 4 & 5 & 4 & 4 & 5 & 5 & 5 & 5 \\
\hline Pastizal inducido & 2 & 3 & 3 & 3 & 2 & 0 & 3 & 3 & 4 & 3 & 3 & 4 & 4 & 4 & 3 \\
\hline Pastizal natural & 3 & 4 & 3 & 3 & 2 & 0 & 3 & 3 & 4 & 4 & 4 & 4 & 4 & 4 & 3 \\
\hline Cuerpos de agua & 1 & 2 & 0 & 4 & 0 & 1 & 1 & 1 & 3 & 2 & 3 & 3 & 4 & 4 & 4 \\
\hline Área urbana & 0 & 1 & 0 & 0 & 0 & 0 & 1 & 1 & 0 & 0 & 1 & 3 & 2 & 2 & 3 \\
\hline
\end{tabular}


capacidades muy bajas o nulas para proporcionar SE de provisión y regulación, mientras que las coberturas naturales tienen alta capacidad para proveer SE. Para los servicios culturales, se encuentra una mayor oferta en paisajes naturales que en áreas urbanas. También se observa que en la cobertura de bosque y matorral existe una mayor capacidad de oferta potencial, debido a que la mayor parte del territorio está dividido en ejidos ${ }^{1}$ y es de difícil acceso. Además de que en la parte alta y media de la microcuenca está dentro del área natural protegida, por lo que aumenta su grado de conservación.

\section{Demanda potencial de SE}

De la encuesta se recopilaron datos de 108 encuestados que habitan principalmente en Jurica y Jurica Campestre, de los cuales sólo el $26 \%$ de los encuestados conoce o sabe de la Zona Occidental de Microcuencas. El 69\% de los encuestados ha sido afectado por inundaciones recientes y el $78 \%$ está dispuesto a pagar para que se realicen obras de conservación, mientras que quienes no está dispuestos señalan que es una de las razones por las que pagan impuestos. Alrededor del 63\% de los encuestados consideran que podrían aportar entre \$100 y \$200MxN (5-10 USD) mensuales y que los SE más valiosos son la regulación de clima y agua, y le siguen en importancia la mejora de la calidad del aire, el control de la erosión y la recreación y el turismo.

La tabla 3 muestra la integración de esta información en la matriz de demanda potencial de SE en los distintos ecosistemas y se puede apreciar que son más altos en

Tabla 3

Matriz de evaluación de la demanda potencial de los servicios ecosistémicos.

\begin{tabular}{|c|c|c|c|c|c|c|c|c|c|c|c|c|c|c|c|}
\hline \multicolumn{16}{|c|}{ DEMANDA POTENCIAL } \\
\hline & \multicolumn{5}{|c|}{ Servicios ecosistémicos de Provisión } & \multicolumn{5}{|c|}{ Servicios ecosistémicos de Regulación } & \multicolumn{5}{|c|}{ Servicios ecosistémicos Culturales } \\
\hline $\begin{array}{l}\text { Usos de suelo } \\
\text { y vegetación } \\
\text { (ecosistemas) }\end{array}$ & 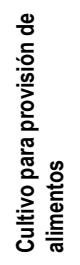 & 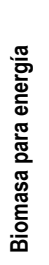 & 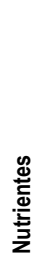 & 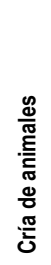 & 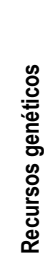 & 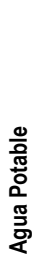 & 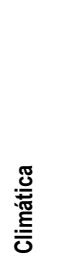 & 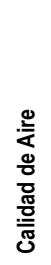 & $\begin{array}{l}\frac{\pi}{3} \\
\frac{9}{6} \\
\frac{0}{0} \\
\circ \\
\frac{0}{3}\end{array}$ & 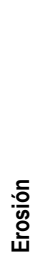 & 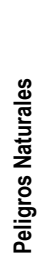 & 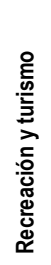 & 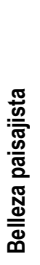 & 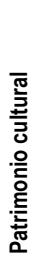 & 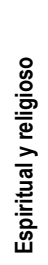 \\
\hline $\begin{array}{l}\text { Agricultura de } \\
\text { temporal }\end{array}$ & 0 & 2 & 3 & 2 & 0 & 1 & 1 & 1 & 2 & 2 & 0 & 0 & 0 & 0 & 1 \\
\hline $\begin{array}{l}\text { Agricultura de } \\
\text { riego }\end{array}$ & 0 & 3 & 4 & 1 & 0 & 3 & 2 & 1 & 3 & 3 & 0 & 0 & 0 & 0 & 0 \\
\hline $\begin{array}{l}\text { Bosque tropical } \\
\text { caducifolio }\end{array}$ & 0 & 0 & 3 & 0 & 0 & 0 & 1 & 1 & 0 & 2 & 0 & 0 & 0 & 0 & 0 \\
\hline $\begin{array}{l}\text { Matorral } \\
\text { crasicaule }\end{array}$ & 0 & 0 & 3 & 1 & 0 & 0 & 1 & 1 & 0 & 1 & 0 & 0 & 0 & 0 & 0 \\
\hline $\begin{array}{l}\text { Matorral } \\
\text { subtropical }\end{array}$ & 0 & 0 & 3 & 0 & 0 & 0 & 1 & 1 & 1 & 1 & 0 & 0 & 0 & 0 & 0 \\
\hline Pastizal inducido & 0 & 0 & 2 & 1 & 0 & 0 & 1 & 1 & 1 & 0 & 0 & 0 & 0 & 0 & 0 \\
\hline Pastizal natural & 0 & 0 & 1 & 0 & 0 & 0 & 1 & 1 & 0 & 0 & 0 & 0 & 0 & 0 & 0 \\
\hline Cuerpos de agua & 0 & 2 & 2 & 3 & 0 & 0 & 1 & 1 & 4 & 0 & 0 & 0 & 0 & 0 & 0 \\
\hline Área urbana & 5 & 5 & 4 & 3 & 4 & 5 & 5 & 5 & 5 & 4 & 5 & 4 & 5 & 4 & 3 \\
\hline
\end{tabular}

Fuente: Elaboración propia (2019).

${ }^{1}$ Ejido: En México es una figura jurídica mediante la cual una comunidad de personas es propietaria de una extensión territorial para repartirla entre sus miembros. 
los ecosistemas dominados por el hombre ya que es donde realizan todas sus actividades (áreas urbanas) y de uso agrícola, debido a que las parcelas son explotadas y no existen prácticas de manejo de suelo ni agua. Los ecosistemas naturales se caracterizan por actividades nulas o con menores demandas.

\section{Balance potencial de SE}

Finalmente, la tabla 4 muestra la matriz correspondiente al balance y se observa una evidente falta de oferta de servicios en los ecosistemas dominados por el hombre (área urbana), mientras que los ecosistemas naturales, especialmente áreas boscosas, se caracterizan por el suministro de diversos servicios que exceden sus demandas, principalmente en biomasa para energía y en todos los SE culturales evaluados.
Tras vincular espacialmente las matrices de evaluación con los ecosistemas, y seleccionar cuatro servicios ecosistémicos representativos de cada tipo de servicio (provisión, regulación y cultural), se obtuvieron cuatro mapas que muestran la oferta, demanda y balance potencial de servicios (Figura 2). En estos se puede observar que existe una oferta medianamente alta en matorrales y bosque, mientras que en la zona urbana es muy baja. En los mapas de demanda, se aprecia que ésta es alta en las zonas urbanas y que en matorrales y bosques es prácticamente nula. $\mathrm{Al}$ hacer el balance entre oferta y demanda potencial, resulta que en matorrales y bosques hay un excedente significativo de oferta potencial de servicios ecosistémicos, mientras que en agricultura es medianamente alto.

Tabla 4

Matriz de evaluación que ilustra el balance potencial de los servicios.

\begin{tabular}{|c|c|c|c|c|c|c|c|c|c|c|c|c|c|c|c|}
\hline \multicolumn{16}{|c|}{ BALANCE POTENCIAL } \\
\hline & \multicolumn{5}{|c|}{ Servicios ecosistémicos de Provisión } & \multicolumn{5}{|c|}{ Servicios ecosistémicos de Regulación } & \multicolumn{5}{|c|}{ Servicios ecosistémicos Culturales } \\
\hline $\begin{array}{l}\text { Usos de suelo } \\
\text { y vegetación } \\
\text { (ecosistemas) }\end{array}$ & 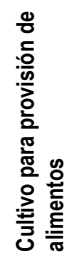 & 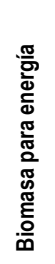 & 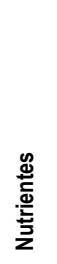 & 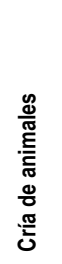 & 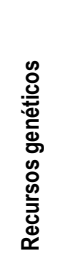 & 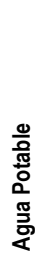 & 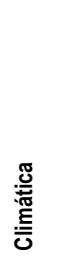 & 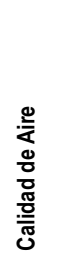 & $\begin{array}{l}\frac{9}{3} \\
\frac{9}{4} \\
\frac{8}{8} \\
\circ \\
\frac{0}{3}\end{array}$ & $\begin{array}{l}\frac{\overline{0}}{\mathrm{o}} \\
\frac{\mathrm{D}}{\mathrm{W}}\end{array}$ & 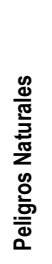 & 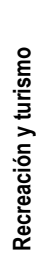 & 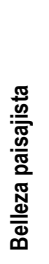 & 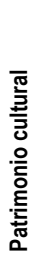 & 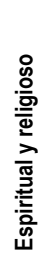 \\
\hline $\begin{array}{l}\text { Agricultura de } \\
\text { Temporal }\end{array}$ & 4 & 1 & 0 & 0 & 1 & -1 & 1 & 0 & 0 & 1 & 1 & 3 & 3 & 4 & 3 \\
\hline $\begin{array}{l}\text { Agricultura de } \\
\text { Riego }\end{array}$ & 3 & -1 & -2 & 1 & 2 & -3 & -1 & 0 & -1 & -1 & 1 & 2 & 3 & 3 & 4 \\
\hline $\begin{array}{l}\text { Bosque Tropical } \\
\text { Caducifolio }\end{array}$ & 1 & 5 & 1 & 3 & 4 & 0 & 4 & 4 & 5 & 3 & 5 & 5 & 5 & 4 & 4 \\
\hline $\begin{array}{l}\text { Matorral } \\
\text { Crasicaule }\end{array}$ & 2 & 5 & 2 & 3 & 5 & 0 & 4 & 4 & 5 & 3 & 4 & 5 & 5 & 5 & 5 \\
\hline $\begin{array}{l}\text { Matorral } \\
\text { subtropical }\end{array}$ & 2 & 5 & 1 & 5 & 4 & 0 & 3 & 3 & 4 & 3 & 4 & 5 & 5 & 5 & 5 \\
\hline Pastizal Inducido & 2 & 3 & 1 & 2 & 2 & 0 & 2 & 2 & 3 & 3 & 3 & 4 & 4 & 4 & 3 \\
\hline Pastizal Natural & 3 & 4 & 2 & 3 & 2 & 0 & 2 & 2 & 4 & 4 & 4 & 4 & 4 & 4 & 3 \\
\hline Cuerpos de Agua & 1 & 0 & -2 & 1 & 0 & 1 & 0 & 0 & -1 & 2 & 3 & 3 & 4 & 4 & 4 \\
\hline Área urbana & -5 & -4 & -4 & -3 & -4 & -5 & -4 & -4 & -5 & -4 & -4 & -1 & -3 & -2 & 0 \\
\hline
\end{tabular}

Fuente: Elaboración propia (2019). 


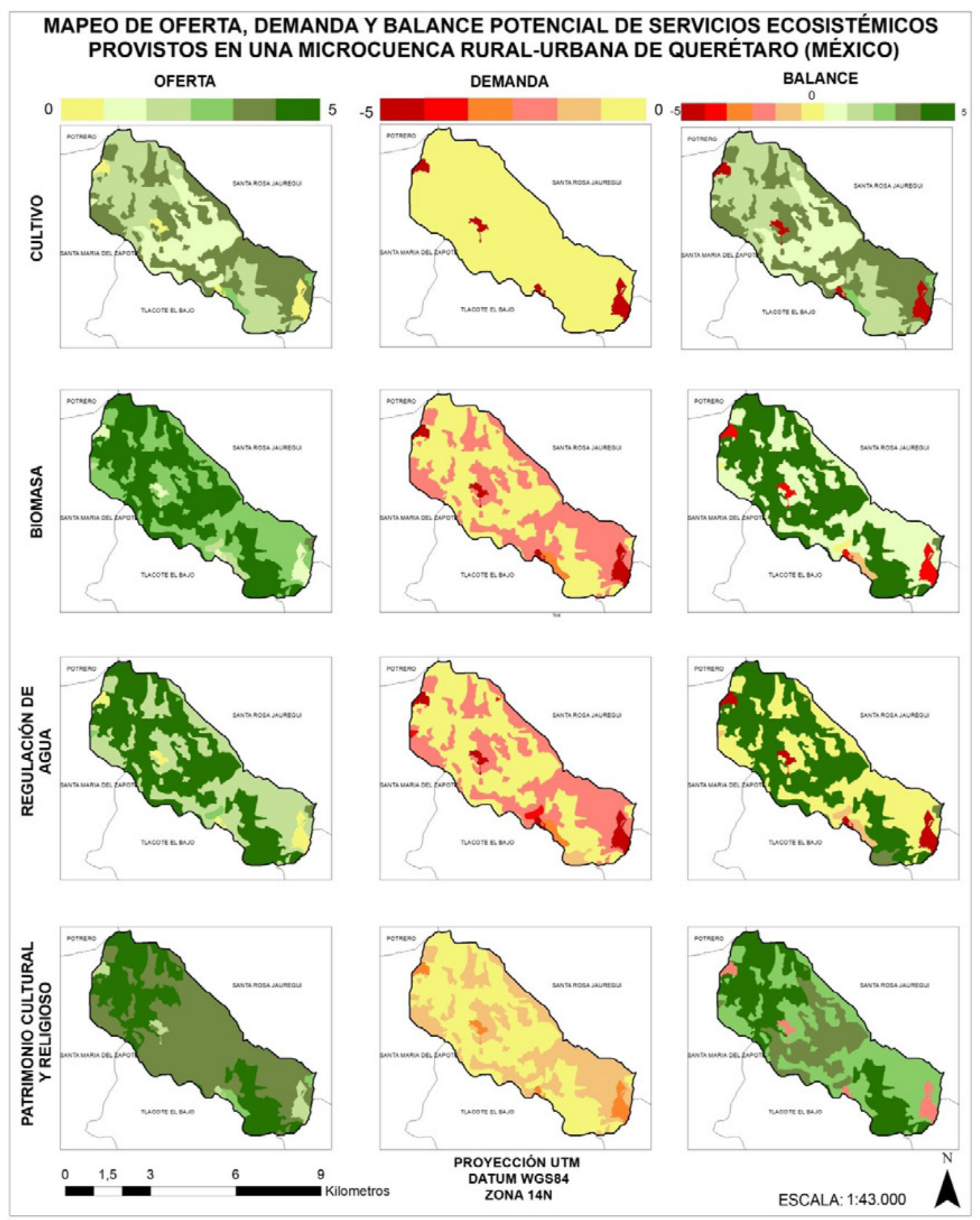

Figura 3. Mapas de evaluación que ilustran la oferta, demanda y balance potencial de los servicios ecosistémicos. Fuente: Elaboración propia (2019). 
Los mapas en la fila izquierda sobre oferta potencial de servicios ecosistémico ilustran las capacidades de los diferentes ecosistemas de la microcuenca para respaldar la integridad ecológica y proveer SE, mientras que los mapas de la fila central representan a la demanda potencial de los seres humanos que viven dentro de la microcuenca y los mapas de la fila derecha ilustran la diferencia que hay en la oferta menos la demanda potencial.

A pesar de tener excedentes de SE en la microcuenca El Nabo donde hay ecosistemas naturales, la zona urbana tiene un alto déficit de servicios, lo que significa que debido a la densidad de población este excedente de oferta es insuficiente para cubrir las necesidades que los habitantes demandan, ya que estos SE no solamente benefician a las personas que habitan dentro de la microcuenca, sino, también a los habitantes cuenca abajo.

\section{Discusión}

El análisis de servicios ecosistémicos basado en la evaluación mediante matrices y mapeo permitió hacer un reconocimiento geográfico sobre la distribución de la oferta, demanda y balance potencial de servicios ecosistémicos actuales. A diferencia de otros trabajos, permitió hacer no solo una estimación de la oferta potencial de SE y su espacialización (p. ej. Kopperoinen et al., 2014; MontoyaTangarife et al. 2017; Arriagada \& Aguayo, 2018;), sino también estimar la demanda potencial y el balance entre ambas. Los resultados son similares a los obtenidos por Burkhard et al. (2012), quienes aplicaron la metodología para determinar la oferta y demanda de energía en una región de Alemania central, revelando los patrones de las actividades humanas en el tiempo y espacio, así como las capacidades de diferentes ecosistemas para proporcionar servicios, sin embargo, mencionan que aún no se aborda el tema de las escalas espaciales y temporales, en este caso la escala espacial en la que se enfoca el estudio es a nivel microcuenca, en cambio sería importante realizar la evaluación de SE a escala temporal, ya que como se mencionó anteriormente, el crecimiento poblacional ha aumentado aceleradamente y ecosistemas podrían ser modificados, por lo que sería importante realizar escenarios a futuro, tanto ideal como negativo.

De acuerdo a Burkhard et al. (2014) mencionan que la oportunidad y la tecnología para proporcionar evaluaciones de SE espacialmente explícitos y balances de oferta y demanda potencial a diferentes escalas ayudan a hacer de los SE una herramienta focal para la gestión ambiental y precisamente es lo que se desea con esta evaluación, que sea útil y de soporte para los tomadores de decisiones.
A su vez, se concuerda con Kopperoinen et al. (2014) donde indican que el principal beneficio de utilizar este enfoque es el ofrecer una plataforma para el debate e intercambio de conocimientos sobre servicios, pero también encontraron desafíos que igual se presentó en este caso de estudio, como la diversidad de servicios, la selección de conjunto de datos dependiendo de la escala, la variación de puntajes dados en cada servicio, y percibir la extensión espacial real del impacto del tema en el potencial específica de oferta de SE

En la microcuenca El Nabo, se recomienda realizar la evaluación planteando escenarios a futuro ideal y negativo, también valorar la oferta de un servicio en particular, identificar la demanda, los principales beneficios, analizar la manera de poder extrapolar los datos a toda la ZOM y determinar si la propuesta de pago por servicios ambientales (PSA) o ecosistémicos es rentable.

Si bien estos análisis pueden ser la base para ubicar el desarrollo de esquemas de pago por servicios ecosistémicos, proporcionan una visión general con precisión limitada, ya que no brindan un valor cuantitativo para la valorización de los servicios, siendo necesario el avanzar para ampliar el número de alternativas de manejo. A su vez, la evaluación de la demanda se ha descuidado hasta ahora en la mayoría de los estudios de SE, tal vez porque es difícil recopilar datos o se centran más en la parte ambiental, dejando aún lado el aspecto social, el cual es importante incluirlo para fomentar el desarrollo de herramientas adecuadas para la toma de decisiones y compensación económica.

\section{Conclusión}

El contar con mapas de oferta, demanda y balance potencial de servicios ecosistémicos representa una contribución importante para aplicaciones futuras e incluso como base para la propuesta de pago por servicios ecosistémicos que se discute en México, y que es tema recurrente en otros países Latinoamericanos. Es importante que estas propuestas no solo se enfoquen a servicios ecosistémicos de importancia global (ej. captura y almacenamiento de carbono), sino también SE de importancia local, como la regulación o prevención de inundaciones, mediada por la capacidad de infiltración y retención temporal de las aguas lluvias que realizan los ecosistemas naturales con más vegetación. Es en este sentido que valorar la demanda es importante. Asimismo, su representación espacial es determinante para fortalecer la dimensión geográfica del territorio, en relación a primero comprender cómo y dónde (ej. parte alta de la microcuenca) se producen procesos ecológicos que tienen beneficios para personas que viven, por ejemplo, 
en la parte baja de una microcuenca y que dependen de la conservación de esos ecosistemas. Más aún, permite detectar necesidades de restauración de ecosistemas degradados que potencialmente podrían ser capaces de proveer más servicios ecosistémicos. Todos estos elementos pueden y deben ser recogidos por los instrumentos de planificación territorial, los que, acompañados de otros instrumentos, por ejemplo, económicos, pueden hacer más factible que la oferta de los servicios ecosistémicos más demandados por la población se mantenga en el tiempo y asegure el bienestar de las personas.

\section{Referencias}

Arriagada, K., \& Aguayo, M. (2018). Mapeo y caracterización servicio ecosistémico de provisión y recreación en la cordillera de Nahuelbuta (37 $30^{\circ} "-38^{\circ} 00^{\prime}$ Sur). Investigaciones Geográficas, (55), 69-88. https://doi. org/10.5354/0719-5370.2018.47024

Balvanera, P., \& Cotler, H. (2007). Acercamientos al estudio de los servicios ecosistémicos. Gaceta Ecológica, 84-85, 8-15.

Balvanera, P., Uriarte, M., Almeida-Leñero, L., Altesor, A., DeClerck, F., Gardner, T., \& Matos, D. M. S. (2012). Ecosystem services research in Latin America: The state of the art. Ecosystem Services, 2, 56-70.

Barzev, R. (2000). Experiencias Replicables de Pago por Servicios Ambientales (PSA) del Recurso Agua en Centroamérica. Recuperado de http://www.bio-nica.info/Biblioteca/BarzevServiciosAmbientales.pdf

Burkhard, B., Kroll, F., Müller, F., \& Windhorst, W. (2009). Landscapes capacities to provide ecosystem services: a concept for landcover based assessments. Landscape Online, 15, 1-22. https://doi.org/10.3097/ 1. 200915

Burkhard, B., Kroll, F., Nedkov, S., \& Müller, F. (2012). Mapping ecosystem service supply, demand and budgets. Ecological indicators, 21, 17-29. https://doi. org/10.1016/j.ecolind.2011.06.019

Burkhard, B., Kandziora, M., Hou, Y., \& Mûller, F. (2014). Ecosystem service potentials flows and demands-concepts for spatial localization, indication and quantification. Landscape online, 34, 1-32. https://doi.org/10.3097/1o.201434
Carpenter S., Mooney, H. A., Agard, J., Capistrano, D., DeFries, R. S., Díaz, S., ...Scholes, R.J., Whyte, A. (2009). Science for managing ecosystem services: Beyond the MEA. Proceedings of the National Academy of Science, 106 (5), 1.305-1.312. https://doi. org/10.1073/pnas.0808772106

CONACYT. (2014). Áreas Naturales Protegidas del Estado de Querétaro. Recuperado de https://www. conacyt.gob.mx/cibiogem/index.php/anpl/queretaro

CONAPO. (2018). Sistema Urbano Nacional 2018.

Recuperado de https://www.gob.mx/conapo/ documentos/sistema-urbano-nacional-2018

Costanza, R., Arge, R., Groot, R. De, Farberk, S., Grasso, M., Hannon, B., ...van den Belt, M. (1997). The value of the world 's ecosystem services and natural capital. Nature, 387, 253-260.

Daily, G. (1997). Nature's Services : Societal Dependence On Natural Ecosystems. Washington D.C: Island Press.

De Groot, R., Wilson, M., \& Boumans, R. (2002). A typology for the classification, description and valuation of ecosystem functions, goods and services. Ecological Economics, 41(3), 393-408. https://doi. org/10.1016/s0921-8009(02)00089-7

De Groot, R.S., Alkemade, R., Braat, L., Hein, L. \& Willemen, L. (2010). Challenges in integrating the concept of ecosystem services and values in landscape planning, management and decision making. Ecological Complexity, 7(3), 260-272. https://doi. org/10.1016/j.ecocom.2009.10.006

De la Barrera, F., Bachman-Vargas, P., y Tironi, A. (2015). La investigación de servicios ecosistémicos en Chile: una revisión sistemática. Investigaciones Geográficas, 50, 3-18. https://doi.org/10.5354/07195370.2015 .41171

Delgado, L. E., \& Marín, V. H. (2015). Ecosystem services: Where on earth?. Ecosystem Services, 14, 2426. https://doi.org/10.1016/j.ecoser.2015.03.002

Dobbs, C., Escobedo, F. J., Clerici, N., De la Barrera, F., Eleuterio, A. A., MacGregor-Fors, I., ...Hernández, H. J. (2019). Urban ecosystem Services in Latin America: mismatch between global concepts and re- 
gional realities?. Urban Ecosystems, 22(1), 173-187. https://doi.org/10.1007/s11252-018-0805-3

European Enviroment Agency (2019). Common International Classification of Ecosystem Services (CICES): Consultation on Version 5.1 Recuperado de https:// cices.eu/

Haines-Young, R., Potschin, M. (2011). Common International Classification of Ecosystem Services (CICES): 2011 Update. Recuperado de https://cices. eu/content/uploads/sites/8/2009/11/CICES_Update_ Nov2011.pdf

Kopperoinen, L., Itkonen, P., \& Niemelâ, J. (2014). Using expert knowledge in combining green infrastructure and ecosystem services in land use planning: an insight into a new place-based methodology. Landscape Ecology, 29, 1.361-1.375. https://doi. org/10.1007/s10980-014-0014-2

Koschke, L., C. Fuerst, S. Frank, S., Makeschin, F. (2012). A multi-criteria approach for an integrated land-cover-based assessment of ecosystem services provision to support landscape planning. Ecological Indicators, 21, 54-66. https://doi.org/10.1016/j. ecolind.2011.12.010

Laterra, P., Barral, A., Carmona, L., Nahuelhual, (2016). Focusing conservation Efforts on Ecosystem service supply may increase Vulnerability of socio-ecological systems. PloS One, 11(5), e0155019. https://doi. org/10.1371/journal.pone.0155019

Millennium Ecosystem Assessment [MEA]. (2005). Evaluación de los Ecosistemas del Milenio Informe de Sintesis. Ecosystems and human wellbeing: synthesis. Washington, D.C.: Island Press.

Montoya-Tangarife, C., De La Barrera, F., Salazar, A., \& Inostroza, L. (2017). Monitoring the effects of land cover change on the supply of ecosystem services in an urban region: A study of Santiago-Valparaíso, Chile. PloS one, 12(11), e0188117. https://doi. org/10.1371/journal.pone.0188117

Pineda, R., Domínguez, M., Hernández, L. Ventura, E. (2005). Microcuencas y Desarrollo Sustentable: tres casos en Querétaro. Universidad Autónoma de Querétaro y Secretaría de Medio Ambiente y Recursos Naturales.
Potschin, M. B., Haines-Young, R. H. (2011). Ecosystem services: exploring a geographical perspective. Progress in Physical Geography, 35(5), 575-594. https://doi.org/10.1177/0309133311423172

Rosas, S. (10 de noviembre de 2014). Queretanos demandan más recursos naturales. El Financiero. Recuperado de https://www.elfinanciero.com.mx/bajio/ queretanos-demandan-mas-recursos-naturales

Sukhdev, P., Wittmer, H., Miller, D. (2014). The Economics of Ecosystems and biodiversity (TEEB): Challenges and Responses. En D. Helm \& C. Hepburn (Eds.), Nature in the Balance, The Economics of Biodiversity (pp.135-150). Oxford: Oxford University Press.

Torres, J. \& Guevara, A. (2002). El potencial de México para la producción de servicios ambientales: captura de carbono y desempeño hidráulico. Gaceta Ecológica, 63, 40-59.

Turner, K., Fisher, B., \& Zylstra, M. (2008) Servicios del ecosistema y teoría económica: integración para la investigación relevante de políticas. Aplicaciones ecológicas, 18, 2.050-2.067. http://dx.doi. org/10.1890/07-1537.1

Vihervaara, P., Kumpula, T., Tanskanen, A., \& Burkhard, B. (2010). Ecosystem services-A tool for sustainable management of human-environment systems. Case study Finnish Forest Lapland. Ecological complexity, 7(3), 410-420. https://doi.org/10.1016/j. ecocom.2009.12.002

Wallace, K. J., (2007). Classification of ecosystem services: problems and solutions. Biological conservation, 139(3-4), 235-246. https://doi.org/10.1016/j. biocon.2007.07.015 